\title{
Formation of amino acid precursors with large molecular weight in dense clouds and their relevance to origins of bio-homochirality
}

\author{
Kensei Kobayashi ${ }^{1}$, Takeo Kaneko ${ }^{1}$, Yoshinori Takano ${ }^{2}$, and \\ Jun-ichi Takahashi ${ }^{3}$ \\ ${ }^{1}$ Graduate School of Engineering, Yokohama National University \\ 79-5 Tokiwadai, Hodogaya-ku, Yokohama 240-8501, Japan \\ email: kkensei@ynu.ac.jp, t-kaneko@ynu.ac.jp \\ ${ }^{2}$ IFREE, Japan Agency for Marine-Earth Science and Technology \\ 2-15 Natsushimacho, Yokosuka 237-0061, Japan \\ email: takano@jamstec.go.jp \\ ${ }^{3}$ NTT Microsystem Integration Laboratories \\ 3-1 Morinosato-Wakamiya, Atsugi 243-0198. Japan \\ email: jitaka@aecl.ntt.co.jp
}

\begin{abstract}
A wide variety of organic compounds have been found in carbonaceous chondrites and comets, which suggests that extraterrestrial organic compounds could have been an important source of the first terrestrial biosphere. In the Greenberg model, these organic compounds in the small bodies were originally formed in interstellar dusts (ISD) in dense clouds by the action of cosmic rays and ultraviolet light. We irradiated a frozen mixture of methanol, ammonia and water with high-energy heavy ions from an accelerator ("HIMAC" in NIRS, Japan) to simulate the action of cosmic rays in dense clouds. Racemic mixtures of amino acids were detected after hydrolysis of the irradiation products. A mixture of carbon monoxide, ammonia and water also gave such complex amino acid precursors with large molecular weights. When such amino acid precursors were irradiated with circular polarized UV light from a synchrotron, enantiomeric excesses were detected. The yield of amino acids was not largely changed between, before, and after CPL-irradiation. The present results suggest that the seed of homochirality of terrestrial amino acids were originally formed in interstellar space.
\end{abstract}

Keywords. Astrobiology, cosmic rays, ISM: dust, ISM: molecules, ultraviolet: stars

\section{Introduction}

Miller (1953) reported that amino acids could be formed in spark discharges in a gas mixture of methane, ammonia, hydrogen and water. Since then, a great number of experiments have been reported to study possible abiotic formation of bioorganic compounds. In earlier studies, strongly reducing gas mixtures such as a mixture of methane, ammonia and water were used as starting materials ("simulated primitive Earth atmospheres"). Many kinds of amino acids were detected in the products when various kinds of energies including ultraviolet light (Sagan \& Khare 1971) and heat (Harada \& Fox 1964). It is suggested, however, that the primitive atmosphere of the Earth was not strongly reduced, but only slightly reduced: Carbon dioxide, carbon monoxide, nitrogen and water were among major constituents of the primitive Earth atmosphere (Kasting 1990). It is not easy to form bioorganic compounds from slightly reduced gases by such major energies 
as ultraviolet light, spark discharge and heat. It is still possible that amino acids and nucleic acid bases can be synthesized from slightly reduced gas mixtures by high-energy particles (Kobayashi et al. 1998), X-rays (Takahashi et al. 1999) or high-temperature plasma (Miyakawa et al. 2002), but the total production rate of bioorganic compounds from slightly-reduced atmospheres are much less than that from strongly-reduced atmospheres.

The other major problems in the origin of life include the riddle of the origin of homochirality of bioorganic compounds, especially amino acids. Proteinous amino acids (except glycine) have D- and L-enantiomers, and both enantiomers can be formed equally when they are abiotically synthesized. On the other hand, terrestrial organisms use only L-amino acids when they synthesize proteins biologically. The development of specific chirality in amino acids has remained one of the most important problems with regard to chemical evolution on the primitive Earth.

A wide variety of organic compounds have been found in materials in carbonaceous chondrites and comets, and the relevance between them and terrestrial origins of life is discussed. Here we discuss possible formation of organic compounds in extraterrestrial environments and show a persuasive scenario for the exogenous origins of homochirality of amino acids by using our recent results of laboratory simulation experiments.

\section{Organic compounds in space}

Carbonaceous chondrites, comets and interplanetary dust particles are three of the major candidates, since they contain considerable amount of organic compounds. Chyba \& Sagan (1992) estimated the inventory rates of organic (or carbon-containing) compounds by meteorites, comets and interplanetary dust particles (IDPs), and concluded that approximately $6 \times 10^{7} \mathrm{~kg}$ of organic carbon was delivered by extraterrestrial bodies.

Organic compounds in carbonaceous chondrites have been comprehensively analyzed. A wide variety of organic compounds have been detected in extracts from carbonaceous chondrites, including amino acids (Kvenvolden et al. 1970) together with unidentified complex organic compounds. Organic globules were found in Tagish Lake meteorite, and isotopic ratio of the carbon suggested that these organic compounds were formed at quite low temperature (10-20 K) (Nakamura-Messenger et al. 2006).

Comets also bring a wide variety of organic compounds. Mass spectra of dusts of Comet Halley (Kissel \& Krueger 1987) suggested that cometary dusts have various types of complex organic compounds including heterocyclic compounds. There were, however, no indications for amino acids in cometary dust. Cometary dust from Comet $81 \mathrm{P} / \mathrm{Wild}$ 2 was captured by Stardust spacecraft. Preliminary analysis of the dusts showed that they have novel types of complex organic compounds that seemed to be more primitive than meteoritic organics (Sandford et al. 2006).

Greenberg proposed a scenario that these exogenous organic compounds were originally formed in interstellar dust (ISD) environments in dense clouds (Greenberg \& Li 1997). Water, carbon monoxide, methanol and ammonia were detected among major constituents of ice mantles of ISDs (Greenberg \& Mendoza-Gomez 1993), but more complex organics could not been observed. A number of studies have been done to see what kind of organic compounds can be formed in such icy environments.

\section{Possible formation of amino acids in ISDs}

Inner dense clouds are as cold as 10-20 K, and most molecules are frozen onto ISDs to form ice mantles. High-energy cosmic rays can go through the dense clouds, and 
ultraviolet light is generated when cosmic rays interact with molecules. Thus, cosmic rays and secondary ultraviolet light are possible energy sources for the reactions in ice mantles of ISDs. When a frozen mixture of molecules found in interstellar dusts such as carbon monoxide and ammonia was irradiated with high-energy protons or UV light, amino acids were detected in hydrolyzed products (Kobayashi et al. 1995, Muñoz Caro et al. 2002, Bernstein et al. 2002). It was difficult, however, to discuss the formation rate of amino acids in ISDs or to characterize the amino acid precursors before hydrolysis since the only limited amount of amino acid precursors were obtained in these experiments.

In order to discuss the energy yield of amino acid precursors, we chose a mixture of methanol, ammonia and water, found in ISD ice mantles as major constituents, and (ii) it is easy to make a large quantity of ice. Energies applied to the materials are highenergy heavy ions, $\gamma$-rays and ultraviolet light. The starting mixture was placed in a liquid nitrogen bath $(77 \mathrm{~K})$, or at ambient temperature so that the starting material was kept as solid ice or liquid, respectively. We performed heavy ion irradiation by using the "HIMAC" accelerator in National Institute of Radiological Sciences (Chiba, Japan). Such ions as helium $\left(150 \mathrm{MeV} \mathrm{amu}^{-1}\right)$, carbon $\left(290 \mathrm{MeV} \mathrm{amu}{ }^{-1}\right)$, neon $\left(400 \mathrm{MeV} \mathrm{amu}^{-1}\right)$ and argon $\left(500 \mathrm{MeV} \mathrm{amu}^{-1}\right)$ were irradiated to the solid $(77 \mathrm{~K})$ or liquid (ambient) mixture of methanol, ammonia and water. Fifty gram each of the mixture was irradiated with heavy ions at the dose rate of 250 - $4800 \mathrm{~Gy} \mathrm{~h}^{-1}$. The total dose was 700 - $15800 \mathrm{~Gy}$, and the total energy deposit was $2.2 \times 10^{20}-4.9 \times 10^{21} \mathrm{eV}$ (Kobayashi et al. 2007). After individual irradiation experiments, an aliquot of the irradiation products was hydrolyzed with $6 \mathrm{M} \mathrm{HCl}$ at $383 \mathrm{~K}$ for 24 hours. Amino acids in the hydrolyzed and unhydrolyzed fraction were analyzed with an ion exchange high performance liquid chromatography (HPLC) system where a post-column derivatization with o-phthalaldehyde and N-acetylL-cystein was applied, and/or gas chromatography / mass spectrometry (GC/MS) after derivatization with ethyl chloroformate and heptafluorobuthanol.

Figure 1 shows mass spectra of glycine derivative: The glycine was obtained by carbon ion irradiation of the frozen mixture of ${ }^{13} \mathrm{C}$-methanol, ammonia and water at $77 \mathrm{~K}$. Besides glycine, proteinous amino acids such as alanine, aspartic acid and non-proteinous amino acids such as $\beta$-alanine, and $\alpha$ - and $\gamma$-aminobutyric acid were detected. In the unhydrolyzed fraction, only small amount of glycine was detected. It showed no free amino acids, but amino acid precursors were formed during irradiation.

The energy yields (G-values) of glycine were determined by irradiation of the mixture with high-energy carbon ions $\left(290 \mathrm{MeV} \mathrm{amu}^{-1}\right)$. The G-values of glycine by irradiation with carbon ions at $77 \mathrm{~K}$ were 0.007 , while that at ambient temperature was 0.014 . The former is about $50 \%$ of the latter. The present results show that amino acid precursors can be formed in ice mantles of ISDs in dense clouds by cosmic rays and/or UV light effectively, even if the temperature of the source mixtures is so low that they are solid ices.

\section{Characterization of complex organic compounds formed from a mixture of carbon monoxide, ammonia and water}

It is proved that complex organic compounds including amino acid precursors can be formed in solid, liquid or gas phase mixtures (Kobayashi et al. 2004a). When a gas mixture of carbon monoxide, ammonia and water was irradiated with high-energy protons, complex organic compounds containing amino acid precursors could be formed quite effectively. The G-value of glycine (after hydrolysis) was as high as 0.23 (Kobayashi et al. 2007). We characterized the complex organic compounds (hereafter referred as CAW). 
Figure $2 \mathrm{a}$ is a gel filtration chromatogram of CAW before acid-hydrolysis. It was shown that the complex organic compounds with amino acid precursors have large molecular weight up to three thousand. Pyrolysis of the product gave acetoamide as a predominant compound, together with many other compounds including polycyclic aromatic hydrocarbons (PAHs: naphthalene, phenanthrene, anthracene, etc.), heterocyclic compounds (imidazole, etc.), and amides (glycolamide, etc.) (Takano et al. 2004). Glycine bound to CAW was much more stable than free glycine against radiation (Kobayashi et al. 2004b).

\section{Possible scenario of the generation of amino acid homochirality}

Amino acids (except glycine and some non-proteinous amino acids) have D- and Lenantiomers and terrestrial organisms use only L-enantiomers to synthesize proteins. The riddle of "the origin of homochirality of biological molecules" has remained one of the most important problems with regard to biochemical evolution. Large numbers of theories regarding the origins of homochirality have been presented (Bonner 1991).

Amino acids found in carbonaceous chondrites were regarded as racemic mixtures. Cronin \& Pizzarello (1997) reported enantiomeric excess of amino acids in the Murchison and Murray meteorites, which suggested that a seed of homochirality of amino acids might have been brought from space to the primitive Earth. Bailey et al. (1998) reported strong IR polarization from the Orion molecular cloud. Several experiments have been conducted to examine the asymmetric photolysis of free amino acids (Nishino et al. 2001). Enantiomeric excesses could be detected only when most of the initial free amino acids were photochemically destroyed.

As described previously, however, no free amino acids, but complex organic compounds containing amino acid precursors were formed in experiments simulating interstellar environments. Thus, we examined the possible emergence of enantiomeric excesses of amino acids by asymmetric photosynthesis of the complex amino acid precursors with CPL.

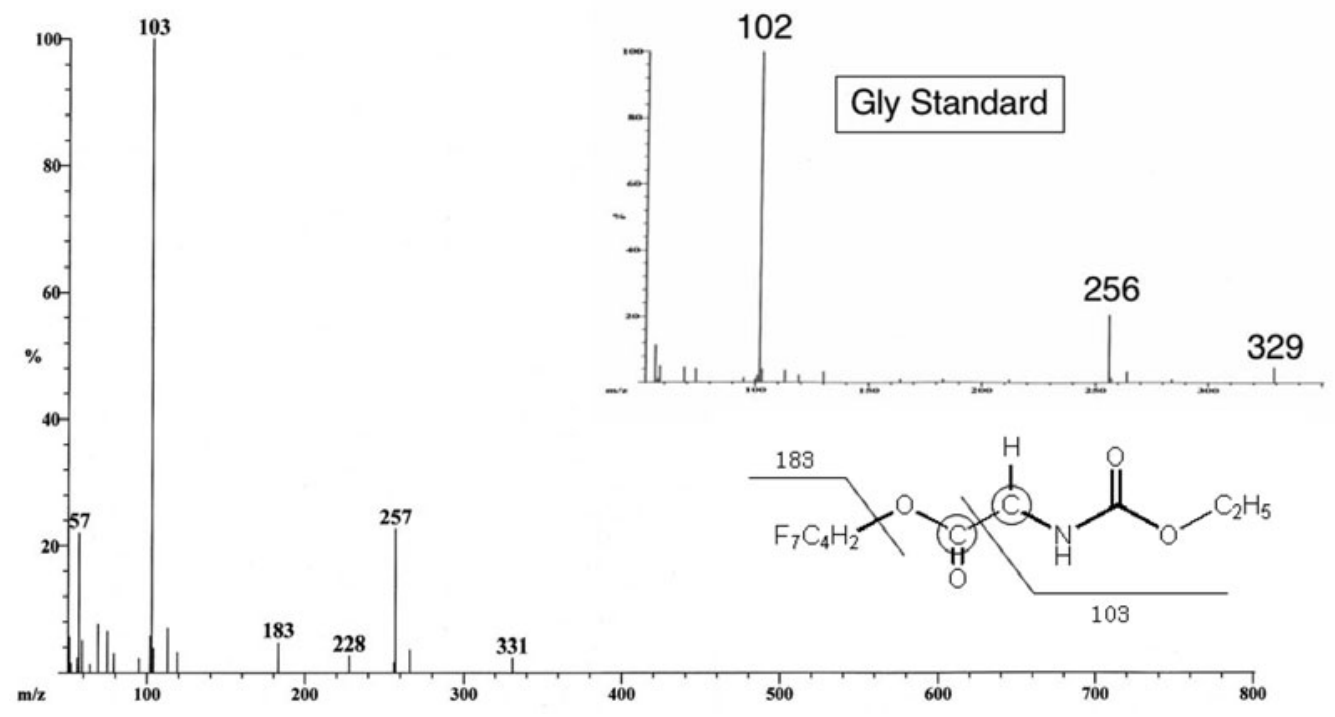

Figure 1. Mass Spectra of glycine synthesized from ${ }^{13} \mathrm{C}$-methanol, ammonia and water with heavy ion irradiation $\left(\mathrm{C}^{6+}, 290 \mathrm{MeV} \mathrm{amu}^{-1}\right)$. The product was hydrolyzed, derivatized and separated by GC before MS analysis. $m / z 331$ is the molecular ion peak of the ${ }^{13} \mathrm{C}_{2}$-labelled glycine derivative, while $m / z 329$ is that of ${ }^{12} \mathrm{C}_{2}$-glycine (standard) derivative. 
(a)
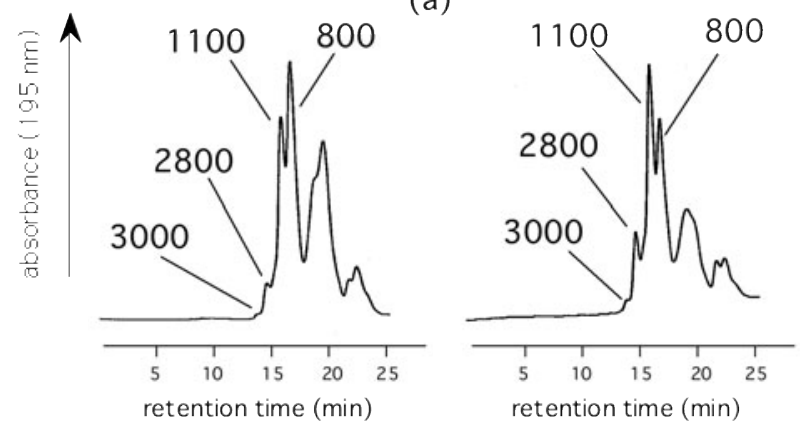

(b)

Figure 2. Gel filtration chromatograms of proton irradiation product from the gas mixtures of carbon monoxide (350 Torr), ammonia (350 Torr) and water (20 Torr). (a) Before CPL-irradiation, and (b) after CPL-irradiation.

Aqueous solution of CAW was subjected to photosynthesis with right (R-) and left (L-) continuous ultra-violet circularly polarized light (UV-CPL; $\lambda>200 \mathrm{~nm}$ ) generated from the ABL-6A beam line of a normal conducting accelerator ring (NAR) at NTT's SR facility.

Figure 2b is a gel chromatogram of CAW after CPL irradiation. Gel filtration chromatograms before and after CPL irradiation had little change. Acid hydrolysis of aliquots of the aqueous solutions before and after CPL irradiation gave almost the same amount of amino acids: Glycine was predominant among the amino acids. These results showed that the complex organic compounds with amino acid precursors are not largely decomposed by CPL-irradiation, while free amino acids were easily decomposed in the same conditions.

Amino acid enantiomers were separated by reverse-phase high performance liquid chromatography (RP-HPLC) after pre-column derivatization with o-phthalaldehyde and Nacethyl-L-cystein. Glycine is achiral, and thus the enantiomeric excess (\% D - \% L) of alanine, which is the predominant chiral amino acid, was precisely determined. D- and L-alanine appeared only after acid hydrolysis, which indicated no free amino acid analog but combined amino acid analog was constituted CPL irradiated sample. Enantiomeric excesses (e. e.; \% D - \% L) of $+0.44 \%$ and $-0.65 \%$ were obtained by R-CPL and L-CPL, respectively (Takano et al. 2007). It is reasonable evidence of asymmetric formation that plus and minus experimental values of enantiomeric excesses occurred by R-CPL and L-CPL, respectively.

There are many other scenarios of the origin of homochirality. Gusev et al. (2007) proposed the following scenario. Polarized electrons produced from neutrons, released in a supernova explosion, can produce molecular asymmetry in dense clouds. We are experimentally examining this hypothesis by using a strong $\beta$-ray source $(50 \mathrm{Ci})$ of ${ }^{90} \mathrm{Sr}$ ${ }^{90}$ Y. Preliminary results were published (Burkov et al. 2008).

If small e.e. of amino acid precursors formed in space was brought to the primitive Earth's ocean, it should have been enlarged. Soai et al. (1995) reported that quite small e.e. could be enlarged to more than $99 \%$ through autocatalytic reactions.

\section{Conclusion}

The present results of laboratory simulation showed that amino acid precursors could be effectively formed in ice mantles of ISDs by cosmic radiation and ultraviolet light, though they are solid ices at low temperature. The amino acid precursors are quite 
complex molecules with large (more than 1,000) molecular weight, and more stable in cosmic environments than free amino acids. It was also suggested that a seed of homochirality of amino acids was generated by some cosmic relevance. Abiotic formation of several types of RNA bases required the presence of reduced and oxidized species in the starting materials. Ice mantles of ISDs are thus promising environments for it. We can summarize that bioorganic compounds endogenously formed in ISDs are significant sources for the first crucial building blocks of life on Earth. They were not sophisticated molecules like ribonucleic acids (RNAs) and proteins, but they could have a seed of biological functions. Thus, we would like to call them Garakuta molecules, i. e., molecules with low performances (Figure 3).

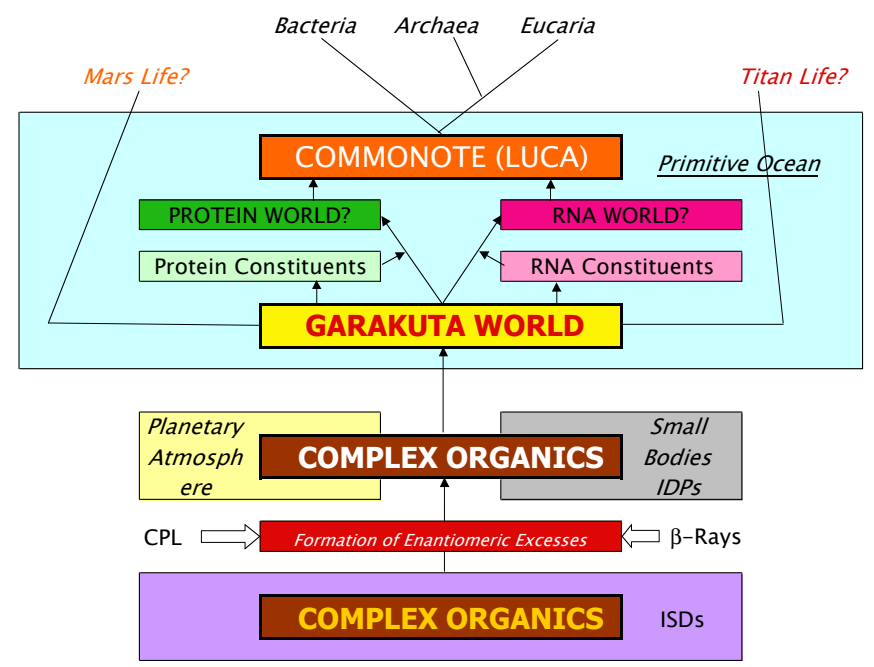

Figure 3. A novel scenario of chemical evolution toward the origin of life. "Garakuta" are molecules with quite low performance.

It is also plausible that these exogenous organics were also supplied to other planets than Earth. If comets can bring sufficient kinds and amounts of bioorganic compounds to planets, xeno-planetary biospheres might be generated, regardless of the composition of primary planetary atmospheres. It will be quite interesting to find and analyze cometary organics as well as organic compounds in interplanetary dusts (IDPs). IDPs have been collected in such terrestrial environments as the stratosphere, ices in Antarctica, and in the deep ocean, but it is quite difficult to analyze bioorganic compounds in them due to possible contamination from the terrestrial biosphere.

We are planning a novel astrobiology mission "Tanpopo" by utilizing the International Space Station (ISS). In the mission, interplanetary dusts will be caught with ultra-low density silica gel (aerogel) placed on the exposure facility of Japanese Experimental Module (JEM) of ISS. In addition, several organic compounds and bacteria will be directly exposed to space environment (including solar extreme-UV light and cosmic rays). The mission is scheduled from 2011.

\section{Acknowledgements}

The authors express their thanks to Dr. Satoshi Yoshida, National Institute for Radiological Studies, Japan, and Dr. Yasuyuki Muramatsu, Gakushuin University, for their kind assistance in heavy ions irradiation. The authors also would like to thank Dr. H. 
Ikeda and Dr. D. Hiroishi, Research Center for Nuclear Science and Technology, University of Tokyo, for their kind help in ${ }^{60}$ Co experiments, and to Dr. Katsunori Kawasaki, Tokyo Institute of Technology for his kind help in proton irradiation experiments. This research was supported in part by a Grant-In-Aid (Nos. 14340170 and 17104050) from MEXT (Ministry of Education, Culture, Sports, Science and Technology, Japan).

\section{References}

Bailey, J. A., Chrysostomou, A., Hough, J. H., Gledhill, T. M., McCall, A., Clark, S., Menard, F., \& Tamura, M. 1998, Science, 281, 672

Bernstein, M. P., Dworkin, J. P., Sandford, S. A., Cooper, G. W., \& Allamandora, L. J. 2002, Nature, 416, 401

Bonner, W. A. 1991, Origins Life Evol. Biosphere, 21, 59

Burkov, V. I., Goncharova, L. A., Gusev, G. A., Kobayashi, K., Moiseenko, E. V., Poluhina, N. G., Saito, T., Tsarev, V. A., Xu, J., \& Zhang, G. 2008, Origins Life Evol. Biosph., 38, 155.

Chyba, C. F. \& Sagan, C. 1992, Nature, 355, 125

Cronin, C. R. \& Pizzarello, S. 1997, Science, 275, 951

Greenberg, J. M. \& Li, A. 1997, Adv. Space Res., 19, 981

Greenberg, J. M. \& Mendoza-Gomez, C. X. 1993, in: J. M. Greenberg, C. X. Mendoza-Gomez \& V. Pirronerllo (eds.), The Chemistry of Life's Origin, (Dordrecht, Kluwer Academic) p. 1

Gusev, G. A., Saito, T., Tsarev, V. A., \& Uryson, A. V. 2007, Origins Life Evol. Biosph., 37, 259.

Harada, K. \& Fox, S. W. 1964, Nature, 201, 335

Kasting, J. M. 1990, Origins of Life, 20, 199

Kissel, J. \& Krueger, F. R. 1987, Nature, 326, 755

Kobayashi, K., Kaneko, T., Saito, T., \& Oshima, T. 1998, Origins Life Evol. Biosphere, 28, 155

Kobayashi, K., Kasamatsu, T., Kaneko, T., Koike, J., Oshima, T., \& Saito, T. 1995, Adv. Space Res., 16, 21

Kobayashi, K., Ogawa, T., Tonishi, H., Kaneko, T., Takano, Y., Takahashi, J., Saito, T., Muramatsu, Y., Yoshida, S., \& Utsumi, Y. 2007, IEEJ Trans. EIS, 127, 293

Kobayashi, K., Takano, Y., Masuda, H., Tonishi, H., Kaneko, T., Hashimoto, H., \& Saito, T. 2004a, Adv. Space Res., 33, 1277

Kobayashi, K., Tonishi, H., Tsuboi, T., Suzuki, N., Kaneko, T., Takano, Y., Hashimoto, H., \& Yamashita, M. 2004b, Biol. Sci. Space, 18, 179.

Kvenvolden, K. A., Lawless, J., Pering, K., Peterson, E., Flores, J., Ponnamperuma, C, Kaplan, I. R., \& Moore, C. 1970, Nature, 228, 923

Miller, S. L. 1953, Science, 117, 528

Miyakawa, S., Yamanashi, H., Kobayashi, K., Cleaves, H. J., \& Miller, S. L. 2002, Proc. Nat. Acad. Sci. USA, 99, 14628

Muñoz Caro, G. M., Meierhenrich, U. J., Schutte, W. A., Barbier, B., Segavia, A., Rosenbauer, H., Thiemann, W. H.-P., Brack, A., \& Greenberg, J. M. 2002, Nature, 416, 403

Nakamura-Messenger, K. Messenger, S., Keller, L. P., Clemett, S. J., \& Zolensky, M. E. 2006, Science, 314, 1439

Nishino, H., Kosaka, A., Hembury, G. A., Shitomi, H., Onuki, \& H, Inoue, Y. 2001, Org. Lett., 3,921

Sagan, C. \& Khare, B. N. 1971, Science, 173, 417

Sandford, S. A., et al. 2006, Science, 314, 1720.

Soai, K., Shibata, T., Morioka, H. \& Choji, K. 1995, Nature, 378, 767

Takahashi, J., Hosokawa, T., Masuda, H. Kaneko, T., Kobayashi, K., Saito, T., \& Utsumi, Y. 1999, Appl. Phys. Lett., 74, 877

Takano, Y., Takahashi, J., Kaneko, T., Marumo, K., \& Kobayashi, K. 2007, Earth Planet. Sci. Lett., 254, 106

Takano, Y., Tsuboi, T., Kaneko, T., Kobayashi, K., \& Marumo, K. 2004, Bull. Chem. Soc. Jpn., 77,779 


\section{Discussion}

Mumma: I was very interested in your comments on mechanisms that might lead to homochirality in meteoritic amino acids. The problem I have with this is that, although they might do so in free space as you have suggested, those amino acids probably didn't form there. Indeed if you go to the CM, CI, and CR meteorites, the number of amino acids dramatically decrease, leading one to wonder whether any amino acids were present in organic material before entering the solar nebula. If you form them in the solar nebula, then the whole issue of circularly polarized light causing this homochirality or enantiomeric excesses, or energetic electrons which you have also suggested, seems to me to not be relevant. So I then pose the question that there must be some other processes that is active in the protoplanetary disk that creates this enantiomeric excess. Perhaps you could comment on this.

KOBAYASHI: Thank you very much for your comments. Yes, my idea is that complex organic acids are formed originally in dense clouds, but they are modified in other environments like diffuse clouds, protoplanetary nebula, and so on. This action could take place in any of these environments. 